\title{
Colombian multicentric study on epidemiology of diabetic ketoacidosis in children with type I diabetes mellitus
}

\begin{abstract}
Background: Type 1 diabetes is a growing disease worldwide, where ketoacidosis is a serious cause commonly resulting in hospitalization. Little is known regarding the epidemiology of the development of ketoacidosis in Colombian children with a known diagnosis of diabetes.
\end{abstract}

Objective: To describe the epidemiological profile of children with type 1 diabetes and ketoacidosis diagnosis.

Settings: Using the online survey, http://www.e-encuesta.com/answer. do? testid $=$ cRetXSIVSLk, seven pediatric intensive care units of the four main cities throughout the country reported epidemiological data of the patients that were admitted with episodes of ketoacidosis other than diabetes debut, between May of 2014 and May of 2016

Measurement and main results: Fifty-two events of diabetic ketoacidosis in 47patients between ages 3 and 17 were included. The patients had mean glycosylated hemoglobin of $12 \%$ (SD:2.9) and the median insulin dose used was $0.91(\mathrm{IQR} 0.74-1.2 \mathrm{UI} / \mathrm{kg}$ ). One hundred percent of patients belonged to middle and low socioeconomic classes, $98.1 \%$ of patients were insured by the health care system, and $27.4 \%$ of patients did not have the necessary supplies for glucose control

Conclusion: The glycosylated hemoglobin levels can evidence that diabetic ketoacidosis patients were generally not well controlled prior to admission. The most common precipitating factors of diabetic ketoacidosis were the low adherence to treatment and infections. Patients must be informed about the importance of adherence to treatment and how to adjust insulin on sick days; the health care system must ensure patients have the supplies necessary for optimal diabetes control.

Keywords: Type 1 diabetes mellitus, diabetic ketoacidosis, risk factors, pediatric intensive care units, epidemiology, multicenter studies
Volume 5 Issue 2 - 2017

\author{
Carolina Jaramillo-Arango,' Yúrika P López- \\ Alarcón, ${ }^{2}$ Juan C Jaramillo-Bustamante, ${ }^{2}$ \\ Mauricio Fernández-Laverde, ${ }^{3}$ Rosalba \\ Pardo-Carrero, Alejandro Marín-Agudelo ${ }^{2}$ \\ 'Pediatric endocrinologist, Grupo de diabetes Hospital Pablo \\ Tobón Uribe, Colombia \\ 2Pediatric intensive care specialist, Hospital General de Medellín, \\ Colombia \\ ${ }^{3}$ Pediatric intensive care specialist, Hospital Pablo Tobón Uribe \\ and Clínica Soma, Universidad CES, Colombia \\ ${ }^{4}$ Pediatric intensive care specialist, Clínica Infantil Colsubsidio, \\ Universidad del Rosario, Colombia
}

Correspondence: Carolina Jaramillo-Arango, Grupo de diabetes, Hospital Pablo Tobon Uribe-Medellín, Calle 78B \# 69240, Colombia,Tel 445-9000,Email cjaramilloarango@gmail.com

Received: May 16, 2017| Published: July 26, 2017
Abbreviations: DKA, diabetic ketoacidosis; HbAlc, glycosylated hemoglobin; ISPAD, international society for pediatric and adolescent diabetes; $\mathrm{Kg}$, kilograms; L, liter; $\mathrm{mEq}$, milliequivalents; PICU, pediatric intensive care unit; T1DM, type 1 diabetes mellitus; IU, international units,

\section{Introduction}

Diabetes in children constitutes a growing diagnosis over the past year that leads to high social and economic costs. ${ }^{1}$ Although the prevalence in our setting remains unknown, it is estimated that approximately $0.26 \%$ of individuals under 20 years have diabetes type 1 or $2 .^{2}$ Diabetic ketoacidosis (DKA), defined as a blood glucose of $>200 \mathrm{mg} / \mathrm{dl}$, ketone presence in the blood, blood $\mathrm{pH}$ of $<7.3$, and serum bicarbonate levels of $<15 \mathrm{mEq} / \mathrm{L}$, is an acute diabetes complication and is potentially life-threatening, resulting from absolute or relative deficiency of circulating insulin and the combined effects of the counter regulatory hormones. ${ }^{3,4}$ Although progress in the handling of DKA has lowered mortality to nearly $2 \% .{ }^{5}$ even today, 90 years after the discovery of insulin, ${ }^{6}$ various studies have demonstrated that the incidence of DKA has not varied over time ${ }^{7,8}$ and that it continues to be the main cause of hospitalization, morbidity, and mortality in children with type 1 diabetes. ${ }^{3,9}$ It has a $7 \%$ prevalence in this population ${ }^{10}$ and is responsible for $8-28 \%$ of hospitalizations among type 1 diabetes (T1DM) patients ${ }^{11}$ and for approximately $50 \%$ of deaths resulting from the disease in diabetic children. Thus, it is important to prevent such DKA episodes in order to reduce morbidity and mortality associated to acute metabolic decompensations in these children. ${ }^{12,13}$

DKA may appear in two contexts in patients with type 1 diabetes: upon disease diagnosis, ${ }^{14,15}$ or development in children with previous diagnosis of diabetes due to poor metabolic control. DKA as a first presentation of diabetes is hard to prevent, except through programs that inform the community of diabetes symptoms ${ }^{16}$ and when the at-risk population is identified. ${ }^{17}$ The prevalence of ketoacidosis in children previously known to be diabetic is much lower than de development of DKA as debut of the disease, studies have shown that it is approximately 8 out of 100 patients per year ${ }^{18}$ and DKA is closely related to diabetes control. In these children, it is necessary to have adequate instruction regarding outpatient treatment, promote treatment adherence, and ensure follow-up through specific diabetes programs for early identification and treatment of metabolic derangement that could prevent the onset of DKA. ${ }^{19}$ 
By the year 2015, the Colombian population amounts to $47,661,787$ million and the coverage of the health system is close to $91 \%$ where $47 \%$ of the population contribute financially to the system and the rest are population subsidized by the state, the prevalence of T1DM in the country is estimated at 3.8 per 100,000 inhabitants. There is evidence that belonging to a family that has little social support, low educational level, low income, lacking health care access, or that is not involved in patient care, especially when they reach adolescence delays in medical care and increase the probability of developing ketoacidosis. This study seeks to describe the clinical and epidemiological profile of patients with diabetic ketoacidosis, which is nonexistent in Colombia, in order to identify the reasons for ketoacidosis development and the socio-demographic factors common to ketoacidosis patients as early identification and prevention could help prevent DKA episodes and thus limit the associated morbidity.

The aim of this study was to determine the clinical and epidemiological profile of children with known T1DM diagnosis that were admitted to intensive care units with diabetic ketoacidosis.

\section{Methods}

\section{Type of study}

Observational, case series study.

Study population: All children and teenagers with type 1 diabetes mellitus with ketoacidosis according to International Society for Pediatric and Adolescent Diabetes (ISPAD) criteria, ${ }^{20}$ admitted to pediatric intensive care units participating in the study were included consecutively.

Sampling: All of the population that met the study eligibility criteria for the stipulated time was included; the unit of analysis used was each diabetic ketoacidosis event developed. Patients were recruited over a 24-month period in which all of the ketoacidosis episodes admitted to pediatric intensive care units were evaluated. Patients with newly diagnosed type 1 diabetes and DKA occurring as the first presentation of the disease where exclude.

Data collection: The pediatric intensive care units in the main cities of Colombia were contacted by phone or e-mail. The project was presented to the ethics committee at each institution, and following approval the participating units filled out an electronic form for each patient that met the inclusion criteria. Since some patients were admitted several times, each ketoacidosis event was included separately. The electronic form was available on this page: http:// www.e-encuesta.com/answer.do?testid=cRctXSIVSLk. Seven pediatric intensive care units participated in the study.

Variables studied: Age at time of diabetes diagnosis, age at time of ketoacidosis, gender, weight at hospital admission, weight when ketoacidosis was resolved, height, insulin schedule, insulin dose, glycosylated hemoglobin (HbAlc) levels, ketoacidosis severity, level of education of caregivers, socioeconomic level, health care coverage, duration of symptoms prior to consultation, supplies (glucose meter, glucose test strips, ketone strips, insulin, injectors), participation in a diabetes care group, number of consultations prior to hospitalization, amongst others.

\section{Statistical analysis}

Qualitative variables were described using absolute and relative frequencies. One-way ANOVA or Kruskal-Wallis tests were used depending on normality and homoscedasticity compliance in order to compare quantitative variables (weight loss evaluated as the difference in weight between weight at time of admission and weight when acidosis was resolved, glycosylated hemoglobin, insulin dose used) according to the degree of decompensation using the D`Agostino \& Pearson, Brown-Forsythe, and Bartlett tests to verify these assumptions. Statistical analysis was conducted using Graphpad Prism, version 6.

\section{Results}

The study included a total of 52 diabetic ketoacidosis events in 47 children with T1DM previously diagnosed from May 2014 through May 2016. Patients were ages 3 to 17, 30 of whom were girls. There was poor diabetes control in $86.5 \%$ of patients as evidenced by $\mathrm{HbA} 1 \mathrm{c}$ between 9.3 and $14 \%$ with a median of $12 \%$. They used intermediate to high insulin doses of $0.74-1.2$ units/Kg with a median of 0.91 units/ $\mathrm{Kg}$. Three patients presented more than one ketoacidosis event, one of whom presented two events and two presented three events. None of the patients died during a DKA episode. Table 1 describes the clinical and demographic characteristics of patients.

Table I Clinical and demographic characteristics of patients

\begin{tabular}{ll}
\hline Characteristic & $\begin{array}{l}\text { Median } \\
\text { (Interquartile } \\
\text { range) }\end{array}$ \\
\hline Age (Years) & $13(10-4)$ \\
Gender M: F & $17: 30$ \\
Diabetes Duration (Years) & $4.5(2.3-6)$ \\
Outpatient Insulin Dose & $0.91(0.74-I .2)$ \\
(IU/Kg of Body Weight) & \\
HbAIc value at time of decompensation (\%)* & $12(3.1)$ \\
Weight loss during decompensation (\%)* & $4.1(4.5)$ \\
\hline Health Care Coverage & $\mathbf{n}(\%)$ \\
\hline Contributory & $5 \mathrm{I}(98.1)$ \\
\hline
\end{tabular}

*Data presented as mean \pm standard deviation

Upon evaluating the socio-demographic characteristics of patients, we found that during hospitalization $23.1 \%$ of children had subsidized health care coverage, $75 \%$ had contributory health care, and $1.9 \%$ ( 1 child) was uninsured. One hundred percent of patients belonged to middle and low socioeconomic classes $(51.9 \%$ lower class and $48.1 \%$ middle class). Regarding the educational level of caregivers in charge of administering patient treatment (ensure adherence to dietary recommendations, carbohydrate count, insulin administration), $46.2 \%$ of caregivers had an elementary school education, $26.9 \%$ had been through secondary school, $13.5 \%$ had technical training, and $5.7 \%$ had a university education.

\section{These were the causes of ketoacidosis identified}

Non-adherence to treatment, $44.8 \%$ of episodes; intercurrent infection, $39.7 \%$ of episodes; trauma or surgical procedure, $3.4 \%$ of episodes. Also, $26.9 \%$ of patients did not have the necessary supplies 
for adequate glycemic control at the time of decompensations. Amongst these supplies, in $23.1 \%$ of episodes, patients did not have glucose test strips, in $11.5 \%$, patients did not have a glucose meter or insulin injectors, in $12.1 \%$, they did not have insulin (ultra-rapid or basal), A single child may have been lacking insulin, insulin injections supplies and glucose strips at the same time. $13.8 \%$ cases had no cause attribution (Table 2).

Table 2 Lack of supplies for glycemic control

\begin{tabular}{ll} 
Supplies & $\mathbf{n ( \% )}$ \\
\hline Lacking Glycemic Control Supplies & $14(26.9)$ \\
Lacking Glucose Meter & $6(11.5)$ \\
Lacking Glucose Test Strips & $12(23.07)$ \\
Lacking Insulin Injectors & $2(3.8)$ \\
Lacking Insulin & $3(5.7)$ \\
Lacking Insulin Injectors And Insulin & $4(7.7)$ \\
Lacking Ketone Strips for Ketoacidosis Diagnosis & $49(94.2)$
\end{tabular}

In 22episodes, patients consulted on the first day of DKA symptoms; in 27episodes, patients consulted within the first week; and in 3 cases, patients had experienced symptoms for over 2 weeks before consulting. Regarding the number of consultations prior to hospitalization, in 27 episodes, patients were hospitalized during the first visit, in 24episodes, patients had had one doctor visit before being hospitalized, and in one case, the patient had visited the doctor twice prior to hospitalization. In $67 \%$ of events, children were not being followed-up by a diabetes care group.

Eighteen patients were diagnosed with mild DKA (blood $\mathrm{pH}$ between 7.2 and 7.3 or bicarbonate between 10 and $15 \mathrm{mEq} / \mathrm{L}$ ), 16 presented moderate DKA ( $\mathrm{pH}$ between 7.1 and 7.2 or bicarbonate between 5 and $10 \mathrm{mEq} / \mathrm{L})$, and 18 patients had severe DKA $(\mathrm{pH}<7.1$ or bicarbonate $<5 \mathrm{mEq} / \mathrm{L}$ ). Table 3 describes the clinical and demographic characteristics of patients according to degree of severity of DKA. No significant differences were found between diabetes duration, outpatient insulin dose, the degree of outpatient glycemic control evaluated with $\mathrm{HbAl}$ c, or in patient weight loss caused by dehydration.

Table 3 Clinical and demographic characteristics of patients based on degree of severity of diabetic ketoacidosis*

\begin{tabular}{|c|c|c|c|c|}
\hline Characteristic & $\begin{array}{l}\text { Mild } \\
\text { Ketoacidosis } \\
n=18\end{array}$ & $\begin{array}{l}\text { Moderate } \\
\text { Ketoacidosis } \\
n=16\end{array}$ & $\begin{array}{l}\text { Severe } \\
\text { Ketoacidosis } \\
n=18\end{array}$ & $\mathbf{p}$ \\
\hline Age(Years)* & $13(9.3-13)$ & II (8-I4) & $13(12-14)$ & 0.36 \\
\hline Diabetes Duration(Years)* & $4(2.8-5)$ & $5(2.5-6.8)$ & $4.5(2-8.3)$ & 0.44 \\
\hline Outpatient Insulin Dose(IU/Kg of Body Weight)* & $I(0.7-1.2)$ & $0.78(0.7-0.97)$ & $I(0.75-I .3)$ & 0.08 \\
\hline $\mathrm{HbAIcValue}$ at time of decompensation(\%) $\dagger$ & $12( \pm 3.5)$ & $\mathrm{II}( \pm 2.9)$ & $12( \pm 2.9)$ & 0.47 \\
\hline Weight Loss During decompensation(\%)† & $3.3( \pm 3.4)$ & $3.9( \pm 4.6)$ & $5.9( \pm 5.3)$ & 0.48 \\
\hline Length of Stay in PICU During Decompensation(Days)* & $\mathrm{I} .5(\mathrm{I}-3.3)$ & $3(1.3-3.8)$ & $2(1.8-3.3)$ & 0.48 \\
\hline
\end{tabular}

*Data presented as median + interquartile range. Kruskal-Wallis test.

tData presented as mean \pm standard deviation. ANOVA test.

\section{Discussion}

Diabetic ketoacidosis is a potentially life-threatening complication and is strongly associated to the quality of patient care. Glycemic control in type 1 diabetes patients requires a strict clinical followup and adequate treatment to prevent short-term complications and reduce the risk of long-term complications, which could result in a substantial increase in the financial impact of the disease.

This is an early if not the initial report utilizing a multi center methodology and electronic reporting to collect systematically data about children with T1DM and DKA. In children with pre-existing type 1 diabetes, $75 \%$ of DKA episodes occur due to failure to administer insulin or due to errors in treatment administration; the remaining cases are the result of inadequate insulin therapy during intercurrent stress processes, such as infections. ${ }^{21-25}$ The primary causes for recurrent DKA in Colombia reflects similar causes in all of our hospital across the Americas and Europe, ${ }^{21-25}$ non-adherence to treatment and intercurrent infections were the main causes for ketoacidosis development in patients.

Despite using a multidisciplinary treatment and follow-up by a diabetes care group, in $33 \%$ of patients, the $\mathrm{HbA} 1 \mathrm{c}$ values of the study population showed an HbAlc mean of $12( \pm 3.1)$, revealing that the patients in the study with diabetic ketoacidosis did not have adequate metabolic control, which is in accordance with other studies suggesting poor glycemic control as the factor associated to ketoacidosis development. ${ }^{25-27}$

Most patients hospitalized due to diabetes decompensations events with ketoacidosis were adolescents with poor glycemic control (median age of 13years, interquartile range 10-14), which agrees with previous studies showing a decrease in glycemic control and increase in insulin resistance evidenced by an increase in outpatient insulin doses administered to the patient ${ }^{28}$ and a decrease in diabetes treatment adherence when patients reach adolescence. ${ }^{26,29}$

Studies in adults have shown how decompensations with DKA in T1DM patients is associated with shorter diabetes duration; ${ }^{27}$ this probably occurs because in the first years of the disease, patients have not yet completely understood their condition or assimilated all the information they receive from their physician; however, unlike these studies, this study observed decompensations episodes both in patients who had recently been diagnosed with diabetes as well as in those who had had the disease for up to 12 years. On the other hand, patient participation in multidisciplinary care groups has helped decrease ketoacidosis incidence as multidisciplinary consultations make it possible to gain a better understanding and self-treatment for diabetes. $^{21,30}$ 
In this study, 39 of the 47 children $(82.1 \%)$ had been diagnosed over 2 years prior to presenting decompensations. This, coupled with the fact that one-third of patients were being followed-up by a multidisciplinary care group in charge of patient care, leads us to the question: What other factors could cause patients to have a poor glycemic control despite having had the disease for several years?

Although we did not have a control group to identify factors associated to decompensations, we explored the presence of some factors reported in the literature associated to decompensations and poor glycemic control, such as the lack of insulin availability and the use of alternative therapies including herbs, prayers, or rituals.

There is also growing evidence of psychosocial factors that may be predictors of DKA episodes. ${ }^{24,31}$ These factors lead to delays in medical care and increase the probability of developing ketoacidosis. ${ }^{32-34} \mathrm{By}$ the year 2015, the Colombian population amounts to $47,661,787$ million and the coverage of the health system is close to $91 \%$ where $47 \%$ of the population contribute financially to the system, ${ }^{35}$ the prevalence of T1DM in the country is estimated at 3.8 per 100,000 inhabitants. ${ }^{36}$ This study had a multicentric focus as it included seven participating pediatric intensive care units. All seven units cared for patients both under contributory and subsidized health care, however, only $1.9 \%$ of patients with DKA were uninsured; all patients belonged to middle and low socioeconomic classes. It is surprising that, despite having health care coverage, $27.4 \%$ of the children did not have the necessary supplies for glycemic control at the time of the decompensations episode; in addition, $94.2 \%$ of them did not have access to ketone measurement, which on sick days can help make an early DKA diagnosis leading to an opportune medical consultation.

Govan L et al. ${ }^{34}$ evidenced that low socioeconomic class and being uninsured by the health care system lead to a higher probability of decompensations with DKA; ${ }^{34}$ the studies carried out in the pediatric population during disease presentation manifested as diabetic ketoacidosis have demonstrated that low income and being uninsured by the health care system are associated to DKA in onset of diabetes. This makes DKA an income inequality marker. ${ }^{32,33}$

We researched whether there were differences amongst patients based on degree of severity of DKA; we found no differences in the HbAlc value associated to the level of decompensations, which is in accordance with previous studies in children and adults. ${ }^{25}$ Likewise, although international guidelines suggest that ketoacidosis severity classified with venous $\mathrm{pH}$ is correlated to the degree of dehydration, ${ }^{3}$ we did not find this correlation in our patients, which agrees with recent studies in children. ${ }^{37,38}$ The separation into the mild, moderate and severe DKA have a little meaning as in others studies, probably because much of this depends on the rapidity of development of dehydration which in the final hours before presentation is success of the child to maintenance of their incomes and outcomes.

We have 52events of ketoacidosis in our study time we consider this is a relevant number since it does not include patients with ketoacidosis who are debuting in diabetes. We are convinced that in an adequate health system, children with diabetes should have all the tools to control their disease and not develop ketoacidosis. Therefore these 52events are relevant to identify these factors and try to impact so that they do not happen again. In this setting, there is no study that explores the epidemiological characteristics of decompensations by diabetic ketoacidosis and we expect this information to help reduce incidence. With the results obtained, we believe that children with T1DM diagnosis and their caregivers must quickly learn to identify the signs and symptoms of diabetes decompensations in order to seek opportune assistance and prevent DKA episodes. The main weakness of this study is not having a comparison group of diabetic children that do not develop ketoacidosis over the course of the disease. This does not allow us to evaluate whether the common factors identified in patients presenting decompensations with ketoacidosis correspond to risk factors for this complication, which would make it possible to take more accurate measures focused on preventing these factors, thus reducing DKA development.

\section{Conclusion}

Information for patients must include instructions on how to adjust insulin dose on sick days and the importance of monitoring ketones during these periods. Also, health personnel must be insistent on the importance of treatment adherence and in trying to recognize difficulties patients may have regarding opportune access to health services and the availability of supplies for glycemic control, which may possibly help decrease diabetic ketoacidosis episodes in children.

\section{Funding details}

None.

\section{Acknowledgements}

The authors would like to thank Libia Maria Rodriguez, bacteriologist and epidemiologist, for her critical analysis of the manuscript.

\section{Declaration of personal interests}

The authors declare no potential conflicts of interest with respect to the research, authorship and publication of this article.

\section{References}

1. Ying AK, Lairson DR, Giardino AP, et al. Predictors of direct costs of diabetes care in pediatric patients with type 1 diabetes. Pediatric Diabetes. 2016;12(3 Pt 1):177-182.

2. https://www.cdc.gov/diabetes/data/statistics/2014StatisticsReport.html

3. Wolfsdorf J, Craig ME, Daneman D, et al. Diabetic ketoacidosis in children and adolescents with diabetes. Pediatric Diabetes. 2009;10(Suppl 12):118-133.

4. Cooke DW, Plotnick L. Management of diabetic ketoacidosis in children and adolescents. Pediatr Rev. 2008;29(12):431-436.

5. Daneman D. Diabetes-related mortality. A pediatrician's view. Diabetes Care. 2001;24(5):801-802.

6. de Leiva A, Brugués E, Leiva-Pérez A. The discovery of insulin: continued controversies after ninety years]. Endocrinología y Nutrición. 2001;58(9):449-456.

7. Curtis JR, To T, Muirhead S, etal. Recent trends in hospitalization for diabetic ketoacidosis in ontario children. Diabetes Care. 2002;25(9):1591-1596.

8. Fritsch M, Rosenbauer J, Schober E, et al. Predictors of diabetic ketoacidosis in children and adolescents with type 1 diabetes. Experience from a large multicentre database. Pediatric Diabetes. 2011;12(4 Pt 1):307-312.

9. Gale EAM. Dying of diabetes. Lancet. 2006;368(9548):1626-1628.

10. Maahs DM, Hermann JM, Holman N, et al. Rates of Diabetic Ketoacidosis: International Comparison With 49,859 Pediatric Patients With Type 1 Diabetes From England, Wales, the U.S., Austria, and Germany. Diabetes Care. 2015;38(10):1876-1882. 
11. Umpierrez GE, Khajavi M, Kitabchi AE. Review: diabetic ketoacidosis and hyperglycemic hyperosmolar nonketotic syndrome. Am J Med Sci. 1996;311(5):225-233.

12. Brink SJ. Diabetic ketoacidosis. Acta Paediatr. 1999;88(427):14-24.

13. Rewers A. Current controversies in treatment and prevention of diabetic ketoacidosis. Adv Pediatr. 2010;57(1):247-267.

14. Morris AD, Boyle DI, McMahon AD, et al. Adherence to insulin treatment, glycaemic control, and ketoacidosis in insulin-dependent diabetes mellitus. The DARTS/MEMO Collaboration. Diabetes Audit and Research in Tayside Scotland. Medicines Monitoring Unit. Lancet. 1997;350(9090):1505-1510.

15. Orlowski JP, Cramer CL, Fiallos MR. Diabetic ketoacidosis in the pediatric ICU. Pediatr Clin North Am. 2008;55(3):577-587.

16. Vanelli M, Chiari G, Ghizzoni L, et al. Effectiveness of a prevention program for diabetic ketoacidosis in children. An 8-year study in schools and private practices. Diabetes Care. 1999;22(1):7-9.

17. Diabetes Prevention Trial-Type 1 Diabetes Study Group. Effects of insulin in relatives of patients with type 1 diabetes mellitus. $N$ Engl J Med. 2002;346(22):1685-1691.

18. Rewers A, Chase HP, Mackenzie T, et al. Predictors of acute complications in children with type 1 diabetes. JAMA. 2002;287(19):2511-2518.

19. Kaufman FR, Halvorson M. The treatment and prevention of diabetic ketoacidosis in children and adolescents with type I diabetes mellitus. Pediatr Ann. 1999;28(9):576-582.

20. Craig ME, Jefferies C, Dabelea D, et al. Definition, epidemiology, and classification of diabetes in children and adolescents. Pediatric Diabetes. 2015;15(Suppl 20):4-17.

21. Dunger DB, Sperling MA, Acerini CL, et al. European Society for Paediatric Endocrinology/Lawson Wilkins Pediatric Endocrine Society consensus statement on diabetic ketoacidosis in children and adolescents. Pediatrics. 2004;113(2):e133-e140.

22. Azoulay E, Chevret S, Didier J, et al. Infection as a trigger of diabetic ketoacidosis in intensive care-unit patients. Clin Infect Dis. 2001;32(1):30-35.

23. Golden MP, Herrold AJ, Orr DP. An approach to prevention of recurrent diabetic ketoacidosis in the pediatric population. $J$ Pediatr. 1985;107(2):195-200.

24. Skinner TC. Recurrent diabetic ketoacidosis: causes, prevention and management. Horm Res. 2002;57(Suppl 1):78-80.

25. Barski L, Nevzorov R, Rabaev E, et al. Diabetic ketoacidosis: clinical characteristics, precipitating factors and outcomes of care. Isr Med Assoc J. 2012;14(5):299-303.
26. Levine BS, Anderson BJ, Butler DA, et al. Predictors of glycemic control and short-term adverse outcomes in youth with type 1 diabetes. J Pediatr. 2001;139(2):197-203.

27. Butalia S, Johnson JA, Ghali WA, et al. Clinical and socio-demographic factors associated with diabetic ketoacidosis hospitalization in adults with Type 1 diabetes. Diabet Med. 2013;30(5):567-573.

28. Cherubini V, Pintaudi B, Rossi MC, et al. Severe hypoglycemia and ketoacidosis over one year in Italian pediatric population with type 1 diabetes mellitus: a multicenter retrospective observational study. Nutrition, Metabolism and Cardiovascular Diseases. 2014;24(5):538-546.

29. Amiel SA, Sherwin RS, Simonson DC, et al. Impaired insulin action in puberty. A contributing factor to poor glycemic control in adolescents with diabetes. N Engl J Med. 1986;315(4):215-219.

30. Ellis DA, Frey MA, Naar-King S, et al. Use of multisystemic therapy to improve regimen adherence among adolescents with type 1 diabetes in chronic poor metabolic control: a randomized controlled trial. Diabetes Care. 2005;28(7):1604-1610.

31. Pawłowicz M, Birkholz D, Niedźwiecki M, et al. Difficulties or mistakes in diagnosing type 1 diabetes in children? - demographic factors influencing delayed diagnosis. Pediatric Diabetes. 2009;10(8):542-549.

32. Mallare JT, Cordice CC, Ryan BA, et al. Identifying risk factors for the development of diabetic ketoacidosis in new onset type 1 diabetes mellitus. Clin Pediatr (Phila). 2003;42(7):591-597.

33. Limenis E, Shulman R, Daneman D. Is the Frequency of Ketoacidosis at Onset of Type 1 Diabetes a Child Health Indicator That is related to Income Inequality? Diabetes Care. 2012;35(2):e5-e5.

34. Govan L, Maietti E, Torsney B, et al. The effect of deprivation and HbA1c on admission to hospital for diabetic ketoacidosis in type 1 diabetes. Diabetologia. 2012;5(9):2356-2360.

35. https://www.minsalud.gov.co/sites/rid/Lists/BibliotecaDigital/RIDE/DE/ PES/informe-congreso-2014-2015.pdf

36. Aschner P. Epidemiología de la diabetes en Colombia. Av En Diabetol. 2010;26(2):95-100.

37. Sottosanti M, Morrison GC, Singh RN, et al. Dehydration in children with diabetic ketoacidosis: a prospective study. Arch Dis Child. 2012;97(2):96-100.

38. Ugale J, Mata A, Meert KL, et al. Measured degree of dehydration in children and adolescents with type 1 diabetic ketoacidosis. Pediatric Critical Care Med. 2012;13(2):e103-e107. 